\title{
Perfil neuropsicológico de um adolescente com síndrome de potocki-lupski (duplicação 17p11. 2 p11. 2): estudo de caso
}

\section{Neuropsychological profile in an adolescent with potocki-lupski syndrome: case study}

\author{
DOI: $10.46919 / \operatorname{arch} v 2 n 5-008$
}

Recebimento dos originais: 01/05/2021

Aceitação para publicação: 31/06/2021

\section{Ariane Cristina Ramello Carvalho}

Doutora em Distúrbios do Desenvolvimento

Instituição de atuação atual: Universidade Presbiteriana Mackenzie

Endereço completo: Rua Sergipe, 401, conjunto 607, Higienópolis - São Paulo - 01243-001

E-mail: ariane.ramello@gmail.com

\section{Sandra de Fátima Barboza Ferreira}

Doutora em Psicologia - PUC-GOIÁS

Instituição de atuação atual: Universidade Federal de Goiás - UFG

Endereço completo: Rua 235 - Setor Universitário - Goiânia - Goiás - CEP 74605-050

E-mail: sandra_barboza@ufg.br

\section{José Salomão Schwartzman}

Doutor em Medicina (Neurologia)

Instituição de atuação atual: Universidade Presbiteriana Mackenzie

Endereço completo: Rua da Consolação, 896 - prédio 28, $1^{\circ}$ andar - Consolação, São Paulo - SP, CEP 01302-907

E-mail: josess@terra.com.br

\author{
Alessandra Gotuzo Seabra \\ Formação acadêmica mais alta: Doutora em Psicologia \\ Instituição de atuação atual: Universidade Presbiteriana Mackenzie \\ Endereço completo: Endereço completo: Rua da Consolação, 896 - prédio 28, $1^{\circ}$ andar - Consolação, \\ São Paulo - SP, CEP 01302-907 \\ E-mail: alessandragseabra@gmail.com
}

\section{RESUMO}

A síndrome de Potocki-Lupski (PTLS), ou trissomia 17p11.2, caracteriza-se por microduplicação da banda 11.2 do cromossomo 17. Trata-se de uma síndrome recém descoberta, associada à síndrome de SmithMagenis - SMS e caracteriza-se pela presença de sintomas dos transtornos do espectro do autismo, comprometimentos cognitivos, dificuldades na fala e aprendizagem, hipotonia e alterações cardíacas congênitas. $\mathrm{O}$ objetivo desse estudo foi estabelecer o perfil cognitivo de um adolescente diagnosticado com PTLS. O participante, do sexo masculino, tinha 19 anos, cursando o nono ano do ensino fundamental, e foi diagnosticado nesta mesma idade, antes desta avaliação, solicitada devido às suas dificuldades de aprendizagem. Foram realizadas entrevista anamnésica, entrevista semi-estruturada com o adolescente, cinco sessões em que foram administrados testes de inteligência (WAIS-III, Raven), de habilidades cognitivas (RAVLT, TDE, D2, FAS) e de personalidade (HTP) e, ao final, realizou-se a entrevista devolutiva com o adolescente e a mãe. Identificaram-se prejuízos importantes de atenção e funções executivas e déficits relacionados à linguagem, com relativa preservação da organização perceptiva-visual 


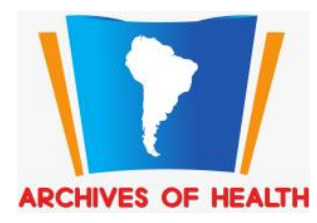

e da velocidade de processamento. $\mathrm{O}$ atraso cognitivo e as dificuldades de aprendizagem, sobretudo relacionadas à aquisição de habilidades escolares, corroboram dados da literatura. Por outro lado, foram observadas pontuações próximas à média em provas de inteligência, o que não reflete sua real funcionalidade, indicando que resultados majorados em testes padronizados podem ser efeito de supertreino ou podem refletir os déficits executivos, usualmente não identificáveis em testes de inteligência. A avaliação neuropsicológica possibilitou o estabelecimento do perfil cognitivo, indicando forças e fraquezas, fornecendo subsídios ao projeto terapêutico no âmbito clínico e educacional.

Palavras-chave: Síndrome de Potocki-Lupski, neuropsicologia, dificuldades de aprendizagem.

\begin{abstract}
Introduction: Potocki-Lupski syndrome (PTLS), or trisomy 17p11.2, is characterized by microduplication of band 11.2 of chromosome 17. It is a newly discovered syndrome, associated with Smith-Magenis syndrome (SMS) and presents symptoms of the autism spectrum disorders, cognitive impairments, speech and learning difficulties, hypotonia and congenital heart alterations. Objective: The aim of this study was to describe the cognitive profile of an adolescent with PTLS. Method: The male participant was 19 years of age, enrolled in the ninth year of elementary education, and was diagnosed at this age, prior to this evaluation, due to his learning difficulties. An anamnesis interview, a semi-structured interview, five sessions in which intelligence tests (WAIS-III, Raven), cognitive ability tests (RAVLT, TDE, D2, FAS) and personality tests (HTP) were administered, and a return interview with the adolescent and the mother were carried out. Results: Significant impairments in attention and executive functions and languagerelated deficits were identified, with relative preservation of the perceptual-visual organization and processing speed. The cognitive delay and learning difficulties, especially related to the acquisition of academic skills, corroborate data from the literature. Scores close to the average in intelligence tests were observed, which did not reflect the real functionality. Conclusions: Higher scores in the standardized tests may have been an effect of intensive training or may reflect executive deficits that are usually unidentifiable in intelligence tests. The neuropsychological evaluation established the cognitive profile, indicating strengths and weaknesses, providing support for the therapeutic project in the clinical and educational context.
\end{abstract}

Keywords: Potocki-Lupski syndrome, cognitive profile, learning difficulties.

\title{
1 INTRODUÇÃO
}

A síndrome de Potocki-Lupski (PTLS), ou trissomia 17p11.2, caracteriza-se por uma microduplicação da banda 11.2 do cromossomo 17. Trata-se de uma síndrome recém descoberta (BROWN, 1996) associada a síndrome de Smith-Magenis (TREADWELL-DEERING; POWELL; POTOCKI, 2010; SHUIB; SAAID; ZAKARIA \& ABDUL-LATIFF, 2017; BISSEL, WILDE, MOSS \& OLIVER, 2018). O diagnóstico depende de mapeamento genético sendo que, preferencialmente, têm sido usadas as técnicas de investigação molecular como a Hibridização in situ por fluorescência - FISH e Hibridização Genômica Comparativa - array-CGH. TREADWELL-DEERING; POWELL; POTOCKI, 2010; GENETIC AND RARE DISEASES INFORMATION CENTER, 2017)

De acordo com Carter et al. (2013), nesta síndrome há presença de características de autismo, dificuldades na fala e de aprendizagem, comprometimentos cognitivos, hipotonia e alterações cardíacas 
congênitas. Apesar de só recentemente a PTLS ter sido caracterizada por meio de diagnóstico sindrômico, há vários relatos clínicos na literatura médica descrevendo as características de pessoas com esta síndrome, especialmente do ponto de vista genético (TREADWELL-DEERING; POWELL; POTOCKI, 2010); contudo há poucos relatos sobre características neuropsicológicas e comportamentais. Segundo TreadwellDeering et al. (2010)., há evidências de atrasos não específicos no desenvolvimento e frequentemente há presença de deficiência intelectual; alterações no sono também são reportadas (PRATICÒ, FALSAPERLA

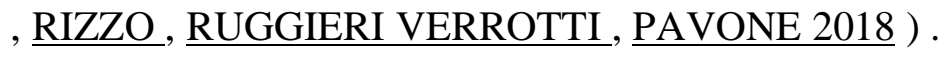

Potocki et al. (2007) realizaram avaliações clínicas multidisciplinares sistemáticas em um subconjunto de 10 indivíduos, incluindo 1 sujeito que abrigou a menor duplicação identificada até aquele momento. Além do atraso do desenvolvimento, encontraram-se deficiência de linguagem e deficiência cognitiva. As características relatadas em mais de 50\% dos pacientes com deleção recíproca de SMS não foram observadas ou foram observadas com pouca frequência na síndrome de duplicação 17p11.2, incluindo estatura baixa, deficiência auditiva, anormalidades otorrinolaringológicas, anormalidades oftalmológicas como miopia e íris hamartomata, anomalias geniturinárias e/ou renais, escoliose clinicamente significativa e hipercolesterolemia. Potocki et al. (2007) sugeriram que a grande maioria dos pacientes com PTLS apresentava características de distúrbio do espectro autista.

De acordo com Neira-Fresnada e Potocki (2015), na infância, frequentemente, a criança com PTLS chega ao atendimento médico em função da hipotonia e dificuldades em obter sucesso em suas intenções. Contudo, a criança mais velha é mais frequentemente identificada por atraso no desenvolvimento, preocupações comportamentais e comprometimento cognitivo. A hipotonia contribui para deficientes habilidades motoras e é um fator que influencia na má alimentação, má articulação e atraso na marcha até 4 anos. O atraso de fala é universalmente observado em PTLS, assim como distúrbios de comunicação como prejuízos na entonação e na prosódia. Cerca de 50\% das crianças estudadas exibiram alguns sinais de apraxia verbal, como dificuldades de planejamento motor e / ou sequenciamento de sons dentro de palavras, e em um subconjunto de crianças possuía dificuldades em alcançar a linguagem verbal (POTOCKI et al. 2007).

Os pacientes que se mostram mais comunicativos geralmente se referem a si mesmos em terceira pessoa, com uma linguagem pedante e com ecolalia imediata ou tardia. A linguagem receptiva também é prejudicada, assim como o desempenho cognitivo, em funções executivas e em comportamentos adaptativos. Os pacientes com PTLS podem usar a comunicação aumentativa como parte de sua linguagem expressiva, implementando a assinatura manual ou outros sistemas como a troca de imagens. Quando submetidos à análise padronizada, a maioria dos indivíduos com PTLS está dentro da faixa moderada de deficiência intelectual, embora alguns situem-se na faixa limítrofe ou não-comprometida. Os déficits de 
funcionamento executivo incluem dificuldades para iniciar atividades, flexibilidade cognitiva e prejuízos em memória de trabalho (POTOCKI; FRESNEDA, 2015).

Segundo Treadwell-Deering, Powell e Potocki (2010), dificuldades comportamentais de vários tipos e gravidade são evidentes em PTLS. Atipicalidade tem sido relatada em 100\% dos pacientes, embora os problemas de atenção, somatização e altos níveis de hiperatividade e ansiedade também tenham sido documentados.

Características dentro do espectro do autismo também são observadas pelos pais e examinadores. Entre as características mais comuns, destacam-se a diminuição do contato visual, dificuldades práxicas, a hipersensibilidade sensorial, os comportamentos repetitivos, a dificuldade de transição, a falta de jogo funcional ou simbólico apropriado e a falta de atenção compartilhada. Em uma avaliação sistemática usando sistemas de pontuação validados, como Autism Diagnostic Interview (ADI-R) e a Autism Diagnostic Observation Schedule-Generic (ADOS-G), cinco de oito crianças preencheram os critérios para o Autism Espectrum Disorder (ASD). Tais características autistas foram observadas em pacientes com duplicação comum, bem como em pacientes portadores de duplicações menores envolvendo o gene RAI1 9, implicando assim este gene sensível à dosagem no fenótipo ASD em PTLS e SMS (POTOCKI; FRESNEDA, 2015).

Para estabelecer a natureza e a extensão de déficits presentes em síndromes, a avaliação neuropsicológica tem sido frequentemente indicada (LEZAK, 1995; LEFÈVRE, 2004). Conforme Miotto (2017), as principais finalidades da avaliação neuropsicológica são contribuir com o diagnóstico diferencial de condições neurológicas e neuropsiquiátricas; inquirir a natureza e o grau de deformações cognitivas e comportamentais; verificar a evolução de quadros neurológicos e psiquiátricos, e propor uma forma de mediação e reabilitação centradas nos prejuízos cognitivos, comportamentais e do dia a dia do paciente. O exame neuropsicológico é um meio de verificação clínica com a finalidade de esclarecer aspectos do funcionamento cognitivo, comportamental e mesmo que não tão aprofundada, mas também de aspectos emocionais de um paciente. Diferenciando de outras investigações cognitivas, a avaliação neuropsicológica parte da premissa de que o comportamento como um todo, o processo cognitivo ou condutas emocionais têm como fundamentos o desempenho de sistemas neurais específicos (Malloy-Diniz et al., 2016). Nesse contexto, diante das evidências ainda escassas sobre características neuropsicológicas relacionadas à PTLS, o presente estudo objetivou traçar o perfil neuropsicológico de um adolescente com síndrome de PotockiLupski.

\section{METODOLOGIA}

Participante: Participou desse estudo um adolescente de 19 anos, sexo masculino, diagnosticado aos 13 anos de idade com deficiência intelectual e encefalopatia não especificada. Mais tarde, aos 19 anos, foi 
diagnosticado com transtorno do espectro do autismo e síndrome de Potocki-Lupski. Tem histórico de várias reprovações escolares e cursava o nono ano do Ensino Fundamental. A queixa principal é relativa às dificuldades de aprendizagem. Conforme relato da mãe durante a entrevista de anamnese, tem dificuldades em todas as matérias escolares, principalmente em matemática. Tem dificuldades de compreender instruções, não compreende metáforas e ironia. É pueril, carinhoso e tem facilidade para fazer amizades.

Instrumentos: Além da entrevista de anamnese, foram utilizados os testes Matrizes Progressivas de Raven-Escala Geral (RAVEN, 2012), que avalia inteligência geral não-verbal, Escala Wechsler de Inteligência para adultos - WAIS-III (WECHSLER, 2004), a qual fornece escores nas escalas verbal e de execução, bem como Quociente de Inteligência (QI) de escala total, QI verbal e QI de execução. Mede o potencial do indivíduo em áreas intelectuais diferentes, como o nível de informação sobre assuntos gerais, e a capacidade de solucionar problemas. Teste de Atenção Concentrada D2 (BRICKENKAMP, 2000), a qual avalia atenção concentrada por meio de uma tarefa de cancelamento. Teste de Aprendizagem Auditivo verbal de Rey (SPREEN; STRAUSS, 1998; MALLOY-DINIZ et al, 2000), para verificar capacidade de memória auditiva verbal, memória de curto e longo prazo, memória de reconhecimento, perseveração, intrusão, susceptibilidade à distratores, bem como a capacidade de retenção de informações após um tempo curto. Teste de Fluência Verbal, Stroop Test, para verificar atenção, capacidade de inibição e funções executivas. Figuras Complexas de Rey-A (OLIVEIRA, RIGONI, 2010), a qual avalia organização, planejamento, julgamento, organização perceptual, orientação visoespacial e função motora.Teste de Desempenho Escolar (STEIN, 2004), o qual avalia habilidades de leitura, escrita e aritmética. Teste de Personalidade House-Tree-Person - HTP (ALVES, TADIVO, 2009), o qual avalia aspectos emocionais e de personalidade.

Procedimentos: Foram realizadas 08 sessões, sendo uma de entrevista anamnésica, uma de entrevista semi-estruturada com o adolescente, cinco sessões de administração dos testes supracitados e, ao final, realizou-se a entrevista devolutiva com o adolescente e com a mãe.

\section{RESULTADOS}

\subsection{INFORMAÇÕES OBTIDAS NA ENTREVISTA DE ANAMNESE:}

O participante é o filho mais novo de uma prole de dois. A mãe relatou intercorrências na gestação (descolamento de placenta), dores e ameaça de aborto. Negou etilismo e tabagismo. O parto foi normal, a termo, cesáreo. O desenvolvimento neuropsicomotor ocorreu tardiamente, demorou para equilibrar o pescoço, apresentou problemas de equilíbrio e coordenação motora, porém começou a andar com 1 ano. Começou a falar com 11 meses e até hoje apresenta dificuldades de articulação das palavras. Foi reprovado no primeiro ano escolar. Tinha dificuldades de aprender o alfabeto e demorou para ler e escrever. Em 2004 foi diagnosticado com deficiência intelectual e logo foi encaminhado para tratamento fonoaudiológico, 
fisioterápico e psicoterápico. Relata que os tratamentos foram benéficos, no entanto, ainda alguns atrasos ainda persistem. A mãe queixa-se das dificuldades para compreender instruções, refere prejuízos em todas as matérias escolares, especialmente em Matemática, relata que o participante não sabe fazer cálculos e tem dificuldade até mesmo na contagem. Relata comportamento pueril, porém organizado, cordato e carinhoso, com facilidade para fazer amizades. Os hábitos de sono são adequados, apresenta hábitos alimentares seletivos e se diz vegetariano.

\subsection{INFORMAÇÕES OBTIDAS DE EXAMES LABORATORIAIS:}

A investigação genética foi feita através da metodologia array - CGH. Foi detectada a seguinte alteração cromossômica em mosaico: arr (hg19)17p11.2 (1685184720217777) x3. RefSeq Genes: A região acima contém mais de 20 genes. Em conclusão, identificou-se um ganho no número de cópias de cromossomo 17 em 17p11.2, abrangendo 3.366 Mb. Esses achados são compatíveis com a síndrome de Potocki-Lupski. A partir da investigação genética o relatório médico atestou a expressão fenotípica também típica da síndrome: Retardo Mental moderado, comportamento autista, dismorfias somáticas e dificuldades de aprendizagem. CID $71.9+$ F.84.0

\subsection{RESULTADOS OBTIDOS NOS TESTES PSICOLÓGICOS}

A avaliação neuropsicológica caracteriza-se pela triangulação de técnicas de observação, entrevista e testagem psicológica. Foram utilizados dois instrumentos para aferir o quociente total de inteligência. O teste de inteligência baseado em fator geral - Teste Raven situou o participante no percentil 21 e na classificação "Intelectualmente abaixo da média". O teste multidimensional WAIS - III indicou inteligência geral em nível médio inferior com perfil disfuncional, indicando maior prejuízo de memória operacional que inclui funções relacionadas à atenção e funções executivas. Observou-se desempenho abaixo da média em linguagem (conforme índice de Compreensão Verbal), porém Quociente de Execução e Velocidade de Processamento dentro da média. A Tabela 1 apresenta os índices fatoriais de Compreensão verbal, Organização perceptiva, Memória operacional e Velocidade do processamento que compõem o Quociente de Inteligência (QI) Total.

Tabela 1. Escala de Inteligência Wechsler para adultos ( $3^{\mathrm{a}}$ Edição) - WAIS-III

\begin{tabular}{|c|c|c|c|}
\hline & WISC III & QUANTIFICAÇÃO & CLASSIFICAÇÃO \\
\hline \multirow{3}{*}{$\bar{\sigma}$} & Verbal & 82 & Média Inferior \\
\hline & Execução & 90 & Média \\
\hline & Total & 85 & Média Inferior \\
\hline \multirow{2}{*}{ 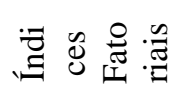 } & Compreensão Verbal & 89 & Média Inferior \\
\hline & Organização Perceptual & 90 & Média \\
\hline
\end{tabular}


As dificuldades atencionais e de funções executivas também foram observadas nos resultados dos testes D2 e Stroop Test, que mostram classificação inferior e prejuízo grave, respectivamente, conforme as Tabelas 2 e 3.

Tabela 2. D2 (Teste de Atenção Concentrada)

\begin{tabular}{cccccc}
\hline D2 & $\begin{array}{c}\text { RESULTADO } \\
\text { BRUTO }\end{array}$ & $\begin{array}{c}\text { TOTAL DE } \\
\text { ERROS }\end{array}$ & $\begin{array}{c}\text { RESULTADO } \\
\text { LÍQUIDO }\end{array}$ & E\% & AO \\
\hline PONTOS & 202 & 21 & 181 & 10 & 13 \\
PERCENTIL & 1 & - & 1 & 10 & 10 \\
\hline
\end{tabular}

Tabela 3. Stroop Test

\begin{tabular}{lccc}
\hline CARTELAS & $\mathbf{1}$ & $\mathbf{2}$ & $\mathbf{3}$ \\
\hline TEMPO ESPERADO & $11 ”$ & $13 ”$ & $21 \%$ \\
TEMPO OBTIDO (segundos) & $21 ”$ & $30 ”$ & 5, \\
$\mathbf{N}^{\mathbf{0}}$ DE ERROS & 0 & 0 & 1 \\
INTERPRETAÇÃO & Prejuízo Grave & Prejuízo Gravíssimo & Prejuízo Gravíssimo
\end{tabular}

O desempenho prejudicado em atividades verbais, além da pontuação inferior no índice de Compreensão Verbal do WAIS-III anteriormente descrito, foi confirmado pelos resultados nos testes RAVLT e FAS, conforme representado no Gráfico 1 e na Tabela 4. 
Gráfico 1. Teste de Aprendizagem Auditivo-Verbal de Rey (RAVLT)

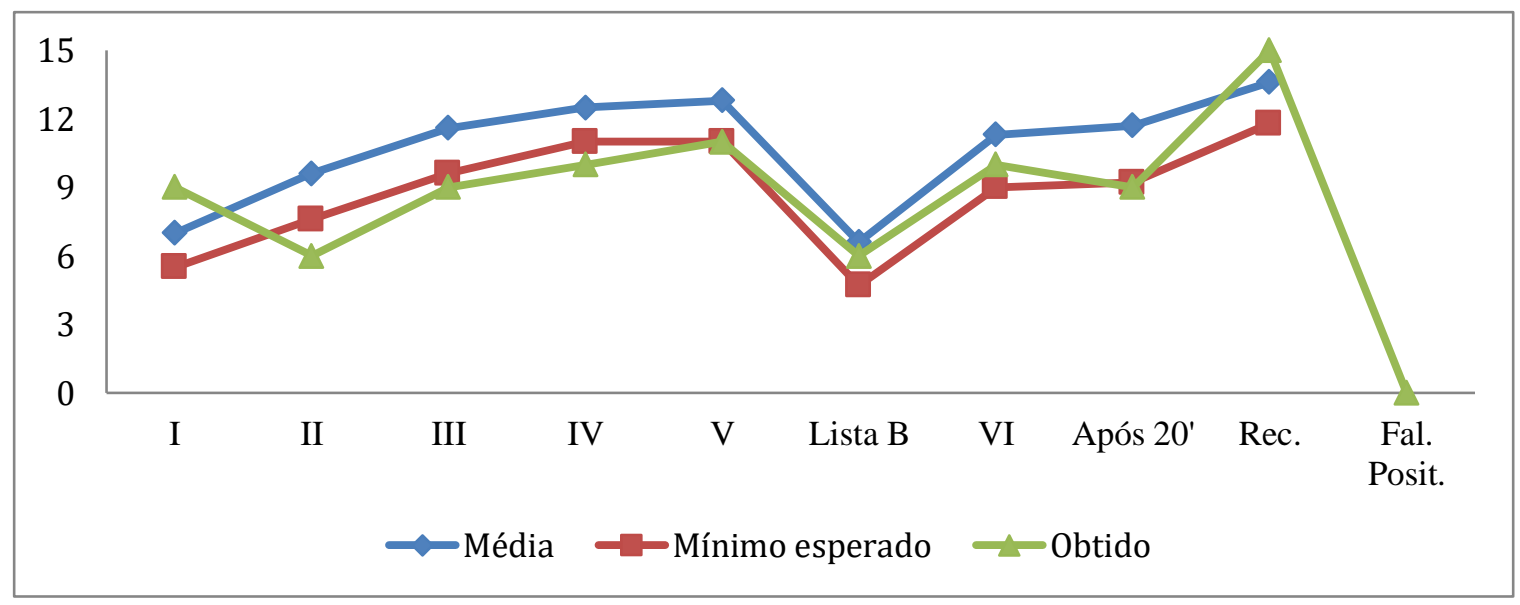

Tabela 4. FAS (Teste de Fluência Verbal -

\begin{tabular}{lcccc}
\multicolumn{1}{c}{ F A S } & OBTIDO & ESPERADO & MÍNIMO & CLASSIFICAÇÃO \\
\hline $\begin{array}{l}\text { FLUÊNCIA } \\
\text { VERBAL }\end{array}$ & 9 & 40,5 & 29,8 & Prejuízo Moderado \\
$\begin{array}{l}\text { FLUÊNCIA } \\
\text { VERBAL }\end{array}$ & 11 & 13 & 13 & Prejuízo Leve \\
SEMÂNTICA & & & & \\
\hline
\end{tabular}

As Tabelas 5 e 6 mostram o resultado obtido em provas de input visual (VOT), avaliado pelo Teste Hooper, e em prova de input visual combinado com função executiva (planejamento e motricidade), avaliados pelo Teste da Figura Complexa de Rey. Os desempenhos confirmam a integridade de funções de organização perceptiva e sugere prejuízo de funções executivas.

Tabela 5. HOOPER (Teste de Organização Visual - Qualitativo)

\begin{tabular}{ccccc}
\hline & OBTIDO & ESPERADO & MíNIMO & CLASSIFICAÇÃO \\
\hline RESULTADO & 20,5 & 25 & 20 & Média \\
\hline
\end{tabular}

Tabela 6. Figura Complexa de Rey

\begin{tabular}{lccc}
\hline REY & RESULTADOS & PERCENTIL & CLASSIFICAÇÃO \\
\hline \multirow{2}{*}{ TEMPO DA CÓPIA } & 20 & 10 & Inferior \\
MEMÓRIA & 3,5 & 100 & Inferior \\
\hline \multicolumn{2}{c}{ Obs.: No tempo da cópia, quanto maior o percentil, pior o desempenho. }
\end{tabular}




\section{DISCUSSÃO}

Os resultados corroboram dados da literatura quanto aos prejuízos cognitivos, comprometimento grave na sustentação da atenção ao longo do tempo, assim como para direcionar o foco atentivo para estímulos relevantes (atenção seletiva), e de linguagem. As dificuldades de aprendizagem são evidentes em função do nível intelectual ser abaixo da média, dos prejuízos atencionais, de funções executivas e de linguagem. Em relação a esta última, ficou flagrante a dificuldade de compreensão verbal e não-verbal, dificuldade para compreender ironia ou mesmo seguir instruções. Além disso, o participante não apresentou nível satisfatório de fluência verbal e, embora conseguisse leitura em voz alta com precisão, não conseguiu ler com fluência, interpretar textos ou mesmo fazer contas básicas de Matemática. Estes resultados inclusive sugerem que os resultados obtidos no teste de inteligência (WAIS-III) sugerem uma performance melhor na avaliação estruturada do que a que de fato o participante apresenta funcionalmente.

De fato, de acordo com Potocki e Fresneda (2015), a linguagem receptiva tende a estar prejudicada, assim como o desempenho cognitivo em funções executivas (iniciar atividades, flexibilidade cognitiva e prejuízos em memória de trabalho) e em comportamentos adaptativos. Além disso, a maioria dos indivíduos com PTLS está dentro da faixa moderada de deficiência intelectual, embora alguns situem-se na faixa limítrofe ou não-comprometida.

Alguns resultados em testes podem ser atribuídos ao supertreino de atividades tendo em vista que o participante passou por várias terapias e tem tido oportunidades de vivenciar um ambiente rico de estimulação. Esse desempenho majorado nos testes fala em favor da pertinência e da qualidade dos programas de reabilitação. No entanto, indicam a limitação na generalização desses processos. Por outro lado, foram observadas pontuações próximas à média em provas de inteligência, o que não reflete sua real funcionalidade, indicando que resultados majorados em testes padronizados podem ser efeito de supertreino ou podem refletir os déficits executivos, usualmente não identificáveis em testes de inteligência

Em relação à memória, o participante apresentou bom desempenho de memória episódica, com curva de aprendizagem ascendente, ou seja, com desempenho crescente conforme era exposto repetidamente à informação; porém, apresentou comprometimento moderado em termos de memória operacional, indicando dificuldade na manipulação mental de diversas informações simultâneas e sequenciais. As memórias semântica e autobiográfica encontraram-se preservadas.

O perfil cognitivo do participante mostrou ainda dificuldade leve de organização perceptiva (investigação, análise e síntese), sendo que ele conseguiu reconhecer diversos objetos tanto reais como impressos, porém apresentou prejuízo leve quanto às capacidades visuoconstrutivas e visuoespaciais. $\mathrm{O}$ participante apresentou prejuízo grave para orientar seu comportamento em direção a metas. O índice de velocidade de processamento foi dentro da média esperada para sua faixa etária de acordo com a WAISIII, porém em todos os outros testes relacionados a esta habilidade revelaram significativa lentidão 
cognitiva. Houve prejuízo grave quanto ao planejamento e refinamento de estratégia na solução de problemas, bem como inflexibilidade cognitiva. As análises clínicas e o desempenho no teste de fluência verbal mostram dificuldade moderada quanto à volição, pois o paciente tendia a quebrar regras e a desistir rapidamente da atividade caso a considere mais difícil. Houve também comprometimento severo para raciocínio lógico e para estabelecer analogias e generalizações. Obteve pontuação extremamente abaixo da média para seu grupo etário no teste de QI não-verbal avaliado pelas Matrizes Progressivas de Raven. Observou-se mais habilidade para informações concretas do que abstratas.

\section{CONSIDERAÇÕES FINAIS}

Este estudo ressalta a importância da realização da avaliação neuropsicológica em pacientes com a síndrome de Potocki-Lupski para estabelecer o perfil cognitivos, as áreas de forças natureza e a extensão de déficits presentes, para direcionar adequadamente, formas de intervenções que possibilitem ao paciente desenvolver habilidades e comportamentos mais favoráveis a aprendizagem e condutas sociais. Este estudo de caso também contribuiu para a compreensão do perfil neuropsicológico em pacientes com a síndrome de Potocki-Lupski (duplicação 17p11.2), uma vez que é uma síndrome recém descrita. 


\section{REFERÊNCIAS}

Bissell, S., Wilde, L., Richards, C., Moss, J., \& Oliver, C. (2018). The behavioural phenotype of PotockiLupski syndrome: a cross-syndrome comparison. Journal of neurodevelopmental disorders, 10(1), 2. doi:10.1186/s11689-017-9221-x

Brickenkamp, R. Teste D2 atenção concentrada. São Paulo: CETEPP, 1992.

Brown, A., Phelan, M. C., Patil, S., Crawford, E., Rogers, R. C., Schwartz, C. Two patients with duplication of 17p11.2: the reciprocal of the Smith-Magenis syndrome deletion? Am. J. Med. Genet. 63: 373-377, 1996. Note: Erratum: Am. J. Med. Genet. 65: 254 only, 1996. [PubMed: 8725788] [Full Text]

Carter, R.D.; Raia, M.; Ewing-Cobbs L. et al. Stress and Well-Being Among Parents of Children with Potocki-Lupski Syndrome. In: Journal Genet Counsel, v.22, 633-642, 2013. https://www.ncbi.nlm.nih.gov/pubmed/?term=Stress+and+Well-

Being+Among+Parents+of+Children+with+Potocki-Lupski+Syndrome

Genetic and rare diseases Information center. (2017) Up Date in Potocki-Lupski Syndrome. https://rarediseases.info.nih.gov/diseases/10145/potocki-lupski-syndrome

Lezak, M. D.; Howieson, D. B.; Loring, D. W. Neuropsychological assessment. 4 ed. New York: Oxford University Press, 2004.

Magoulas PL, Liu P, Gelowani V, Soler-Alfonso C, Kivuva EC, Lupski JR, Potocki L. Inherited dup(17)(p11.2p11.2): expanding the phenotype of the Potocki-Lupski syndrome. Am J Med Genet A. February, 2014; 164A (2):500-504. https://www.ncbi.nlm.nih.gov/pubmed/24311450

Malloy-Diniz, L. F., Mattos, P., Abreu, N., Fuentes, D. (2016). O Exame Neuropsicológico: O que é e para que serve?. Em L. F. Malloy-Diniz, P. Mattos, N. Abreu, D. Fuente. (Orgs) Neuropsicologia: Aplicações Clínicas. (pp. 21-34). Porto Alegre: Artmed.

Miotto, E. C. (2017). Avaliação Neuropsicológica e Funções Cognitivas. Em E. C. Miotto, M. C. S. Lucia, M. Scaff (Orgs). Neuropsicologia Clínica. (pp. 3-31. 2.) Rio de Janeiro: Roca.

Nascimento, E. /Weschsler. (2013) Escala de Inteligência para Adultos - WAIS-III. Adaptação brasileira - 3 ed. São Paulo: Casa do Psicólogo.

Neira-Fresnada, J; Potocki, L. Neurodevelopmental Disorders Associated with Abnormal Gene Dosage: Smith-Magenis and Potocki-Lupski Syndromes. J. Pediatr. Genet. Sep;4(3): 159-167. Published on line 2015 https://www.ncbi.nlm.nih.gov/pmc/articles/PMC4918721/

Oliveira, M.S. Figuras Complexas de Rey - Teste de Cópia e de Reprodução de Memória de Figuras Geométricas Complexas. Adaptação Brasileira. São Paulo, 2010.

Potocki, L., Bi, W., Treadwell-Deering, D., Carvalho, C. M. B., Eifert, A., Friedman, E. M., Glaze, D., Krull, K., Lee, J. A., Lewis, R. A., Mendoza-Londono, R., Robbins-Furman, P., Shaw, C., Shi, X., Weissenberger, G., Withers, M., Yatsenko, S. A., Zackai, E. H., Stankiewicz, P., Lupski, J. R. Characterization of Potocki-Lupski syndrome $(\operatorname{dup}(17)(\mathrm{p} 11.2 \mathrm{p} 11.2)$ and delineation of a dosage-sensitive critical interval that can convey an autism phenotype. Am. J. Hum. Genet. 80: 633-649, 2007. https://www.ncbi.nlm.nih.gov/pmc/articles/PMC1852712/ 
Praticò AD, Falsaperla R, Rizzo R, Ruggieri M, Verrotti A, Pavone P. (2017). A New Patient)with PotockiLupski Syndrome: A Literature Review. J Pediatr Genet. Mar;7(1):29-34. doi: 10.1055/s-0037-1604479. Epub 2017 Jul 27.

Raven, J.C. (2012) Matrizes Progressivas: escala geral. Adaptação brasileira - 5 a ed. Rio de Janeiro: CEPA.

Treadwell-Deering, D.E., Powell, M.P., Potocki, L. Cognitive and Behavioral Characterization of the Potocki-Lupski Syndrome (Duplication 17p11.2). (J Dev Behav Pediatr 31:137-143, 2010). https://insights.ovid.com/pubmed?pmid=20110824

Shuib,S; Saaid, N.N.; Zakaria,Z.; Ismail, J.; Abdul-Latiff, Z. Duplication 17p11.2 (Potocki-Lupski Syndrome) in a child with developmental delay. Case Report. Malaysian J Pathol 2017; 39(1) : 77 - 81. http://www.mjpath.org.my/2017/v39n1/Potocki-Lupski-syndrome.pdf

Stein, L. M. (2004). TDE - Manual para Aplicação e Interpretação. São Paulo: Casa do Psicólogo. 